\title{
Direct growth of single-walled carbon nanotubes on substrates
}

\author{
PENG Fei, LIU Yu, CUI RongLi, GAO DongLiang, YANG Feng \& LI Yan* \\ Beijing National Laboratory for Molecular Sciences, National Laboratory of Rare Earth Material Chemistry and Application, Key Laboratory for \\ the Physics and Chemistry of Nanodevices, College of Chemistry and Molecular Engineering, Peking University, Beijing 100871, China
}

Received October 29, 2011; accepted November 15, 2011; published online December 8, 2011

As one of the most promising candidate material for next generation electronic devices, the reliable and controllable synthesis of high quality single-walled carbon nanotubes (SWNTs) has long been an essential and important issue in the field. Direct growth of SWNTs on flat substrates by chemical vapor deposition (CVD) process is the best way to obtain SWNTs because it is immediately ready for building nano-devices. The orientation of the SWNTs has been well controlled by gas flow or substrate lattice during the CVD growth process. The chirality and structure control of SWNTs is still a big challenge. However, the conductivity selective growth has already partially succeeded. New catalysts have been explored to obtain SWNTs of higher quality. Along with the further progress in the study of the SWNT growth, the precise control over the orientation, position and conductivity of SWNTs is expected to meet the requirements of carbon-based nanoelectronics.

single-walled carbon nanotubes, chemical vapor deposition, selective growth

Citation: $\quad$ Peng F, Liu Y, Cui R L, et al. Direct growth of single-walled carbon nanotubes on substrates. Chin Sci Bull, 2012, 57: 225-233, doi: 10.1007/s11434011-4919-8

Since Iijima et al. [1] observed SWNTs under an electronic microscope in 1993, SWNTs have attracted wide attention because of their unique electrical, chemical, optical, and mechanical properties [2]. One of the most prospective applications is in the area of nano-scaled electronic devices. There have been many reports showing the individual SWNT field-effect transistors (FETs) have good performance [3-5]. However, the success of carbon nanotubebased nanoelectronics highly depends on the supply of SWNTs with designed properties and patterns. SWNTs are normally obtained in bulk form. They need to be purified and dispersed onto the substrates before building devices. This kind of samples are neither suitable for fabricating high performance devices nor for assembling large-area circuits. Because damages and contaminates are unavoidably introduced into SWNTs during the purification and dispersing processes and the locations of SWNTs cannot be controlled accurately on the substrates. Therefore direct growth of SWNTs on substrate by chemical vapor deposition (CVD) is the most suitable approach to obtain SWNTs

*Corresponding author (email: yanli@pku.edu.cn) as the materials for superior electronics. Here, we present part of the recent progress in the direct growth of SWNTs on substrates by CVD processes, including orientation control, the chirality control and the catalysts for CVD growth of SWNTs.

\section{Orientation control}

Applications in nanoelectronics require oriented long SWNTs grown on substrates. Thus a large number of devices can be made from the patterned SWNT arrays in a pre-designed and scalable process. In recent years, several kinds of strategies have been developed to control the growth direction of SWNTs. Among the developed strategies, the gas flow guided growth and the substrate lattice oriented growth both are very effective and flexible therefore the most commonly used methods.

\subsection{Gas flow directed growth}

Using the feeding gas flow to control the orientation of 
SWNT arrays is a promising approach. Liu and co-workers [6] first developed this method. They used a so-called fast-heating CVD method, which heats the substrate very quickly by pulling the substrates with catalyst particles into the pre-heated furnace, to reduce the interaction between catalytic particles and substrates. Therefore, the gas flow can easily lift the catalyst particles above the surface of the substrate. Consequently, the SWNT grows and floats along with the gas flow like a flying kite. SWNT arrays grown following this "Kite-Mechanism" are parallel to the flow direction of the feeding gas with the length in centimeter scale. In the fast-heating method, the key procedure is transferring the substrate to the central zone of the preheated furnace to heat the substrate to the reaction temperature in a very short period. A convection flow is formed in this process because the substrate and the surrounding gas are heated at different speeds and have different temperatures at the initial stage. Such convection flow can lift up some of the catalyst nanoparticles with a nanotube tail. Thus the growing nanotubes are carried by the gas flow and aligned along the direction of the feeding gas during the growth process. Fast-heating is a useful technical to obtain horizontally aligned SWNT arrays.

However, the gas flow is normally as high as hundreds to thousands of sccm (standard cubic centimeter per minute) in fast-heating process to lift up the catalyst nanoparticles for SWNT growth. It is neither economic nor environmentfriendly/green to use such a high gas flow. What's more important, turbulence is much easier to be introduced into the reaction system under the large gas flow, which is very harmful for the steady floating and growth of the SWNTs. Therefore, the length of SWNTs is limited and it is very difficult to obtain large scale SWNT arrays using fast heating method. We [7] developed an ultralow gas flow CVD process to solve this problem. Fast heating is not needed in this process. In comparison to the normally used $1000 \mathrm{sccm}$ for fast heating method, the gas flow is reduced to as low as $1.5 \mathrm{sccm}$ in our strategy. The obtained SWNTs are well oriented with the length as long as several to tens of centimeters (Figure 1(a)). We found that the gas flow rates of
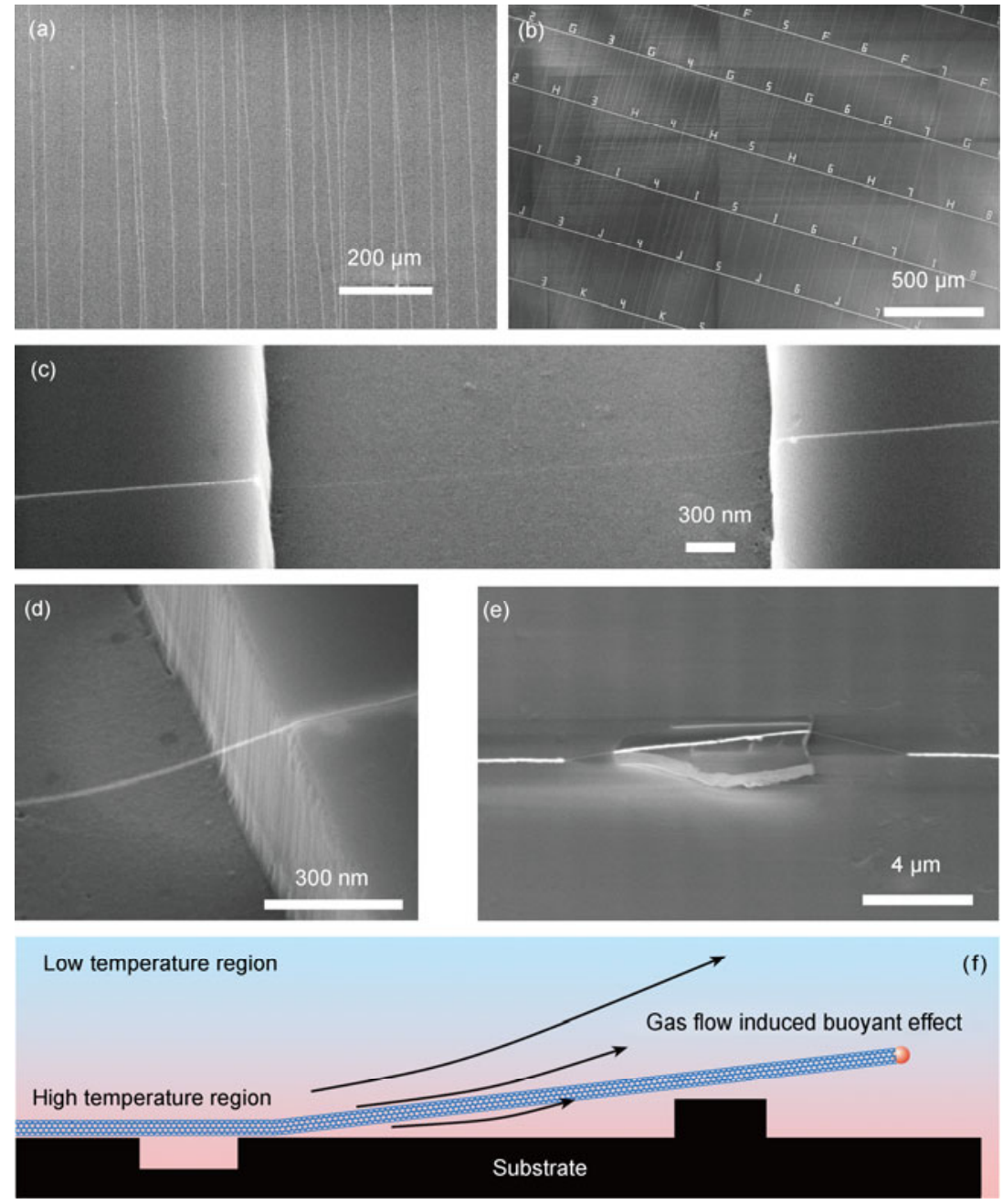

Figure 1 (Color online) (a) Scanning electron microscopy (SEM) image of the horizontally aligned SWNT array grown under ultralow gas flow. (b) SEM image of SWNTs grown across trenched substrate. (c) A suspended SWNT crossing over a microtrench. (d) SEM image of the end of a suspended SWNT crossing over a microtrench. (e) SEM image of SWNTs grown over a micro-obstacle. (f) Schematic drawing of the growth mechanism under ultralow gas flow [7]. 
1.5-150 sccm are suitable for the growth of SWNT arrays in a one inch quartz tube reactor as far as the $\mathrm{CH}_{4}: \mathrm{H}_{2}$ ratio is fixed to $1: 2$. The optimal growth parameter is $970^{\circ} \mathrm{C}, 2.0$ sccm $\mathrm{CH}_{4}$ and $4.0 \mathrm{sccm} \mathrm{H}_{2}$. As shown in Figure 1(b)-(e), the SWNTs can grow across the trenches of $\sim 3 \mu \mathrm{m}$ and climb over the barriers of $\sim 10 \mu \mathrm{m}$ on the substrate surface. This indicates that the SWNTs are still floating in the gas flow during the growing process even at such a low feeding gas flow.

Then, how does the ultralow feeding gas flow method works? The buoyant effect takes the key role in this process. Due to the radiant heat transfer from the heavily heated reactor tube wall to the substrate, the temperature difference between the substrate and the surrounded gas flow which reaches the central zone from a relatively cool front region will generate a vertical gas density difference. Such gas density gradient induces a convection flow which lifts up the nanotubes from the substrate and the nanotubes are consequently aligned by the shear flow nearby the substrate. The Richardson number $R i=\Delta \rho g h / \rho v^{2}$ describes the buoyancy- to-inertia ratio of the mixed convective gas flow. The buoyant force will increase with the decrease of gas flow velocity. The laminar and stability feature of the gas flow can be evaluated by Reynolds number $R e=\rho v d / \mu$. It is obvious that the lower flow velocity also benefits the formation of stable laminar flow. The above two issues both show that it is the low gas flow instead of the high gas flow that is favorable for the lifting and floating of the catalytic nanoparticles and SWNTs. An additional benefit of ultralow gas flow is the remarkable reduction of secondary flow. In the heated furnace tube, the gas near the tube wall is hotter than the gas at the center of the tube. Thus the hotter gas levitates and the cooler gas descends down, forming a symmetrical gas circulation called secondary flow. This secondary flow is unfavorable for the steady floating of SWNTs therefore is bad for growing long SWNTs. However, in the ultralow gas flow method, the secondary flow is much weaker because the low velocity gas flow can be heated more equally. Figure 1f illustrates the mechanism of our ultralow feeding gas flow method for growing large scale SWNT arrays.

Both fast-heating method and ultralow gas flow method can obtain horizontally aligned SWNT arrays. The key of the two strategies is lifting up the nanotubes from the substrates so that they can be guided and aligned by the gas flow. Unidirectional gas flow is used to guide the growth in both the two strategies, so that straight SWNT arrays can be obtained. Non straight SWNT arrays can also be fabricated using the gas flow guided method [8]. When we introduced some barriers to modify the gas flow patterns, we obtained curved SWNTs. As shown in Figure 2, the shapes of SWNTs exactly replicate the streamline patterns. Therefore, we can obtain SWNT arrays with designed pattern by alternating the gas flow streamline using designed gas flow modifiers. This result proves again that gas flow directed growth is a convenient and reliable method to control the SWNTs' growth direction.

\subsection{Substrate directed growth}

Horizontally aligned SWNT arrays can be grown on the crystalline substrate surfaces like sapphire and quartz, using atomic step or substrate lattice as the guiding templates (Figure 3 (d)) [9-11]. Joselevich et al. [9] reported the aligned nanotubes grown on annealed miscut c-plane sapphire. After annealing in the air, the c-plane miscut sapphire surface formed some faceted nanosteps (Figure 3(a)). Such faceted nanosteps could template the formation of periodic SWNT arrays. Zhou and co-workers [10] discovered that they could also obtain well aligned SWNT arrays on a-plane and r-plane with negligible miscut sapphire without atomic nanosteps. By adjusting the density of catalysts, the tube density can be increase to $40 \mathrm{SWNTs} / \mu \mathrm{m}$. They assumed that the interaction between the nanotubes and/or catalyst particles with the substrate contributes to the nanotube alignment (Figure 3(b)). Ding et al. [11] also found that highly aligned SWNT arrays were obtained on quartz substrates with no obvious atomic steps. When different kinds of metal catalysts were used, aligned SWNTs can all formed on quartz substrates with no atomic steps, though the interactions between quartz and the catalyst nanoparticles are different for different types of metal catalysts [12]. So it seems that the catalyst-substrate interaction is not responsible for the alignment of SWNTs. Therefore they proposed that it is the carbon shell precipitated on the outer surface of the catalysts that interacts with the substrate lattice and leads the growth direction of SWNTs. Combining the gas flow direction and the crystalline substrate direction can obtain serpentine nanotubes. Yao et al. [13] designed a specific growth setup to realize a gradual and controllable cooling of samples after switching off the carbon source (Figure 3(c)). When cooling from 975 to $775^{\circ} \mathrm{C}$, the already grown and floated nanotubes by buoyant force fall down to the quartz substrate. Compared to the long nanotubes interacting with the substrate, the small catalyst particle interacting with the substrate is negligible. This proves that the nanotube-substrate interaction is responsible for the SWNTs' alignment.

Gas flow directed growth and substrate oriented growth both have their own advantages. Gas flow directed strategy has no preference on substrates. SWNT arrays can be fabricated on all kinds of flat substrates. Especially, we can use this method to grow SWNT arrays on silicon wafers with silicon oxide layer on top, which is well compatible with current silicon-based microelectronics technology. In addition, gas flow directed growth can obtain SWNTs with much larger lengths (centimeters, or even longer), which is superior for building large scale integrated circuits. The substrate directed growth can obtain SWNT arrays of much higher densities. And the formation of multi-walled carbon 

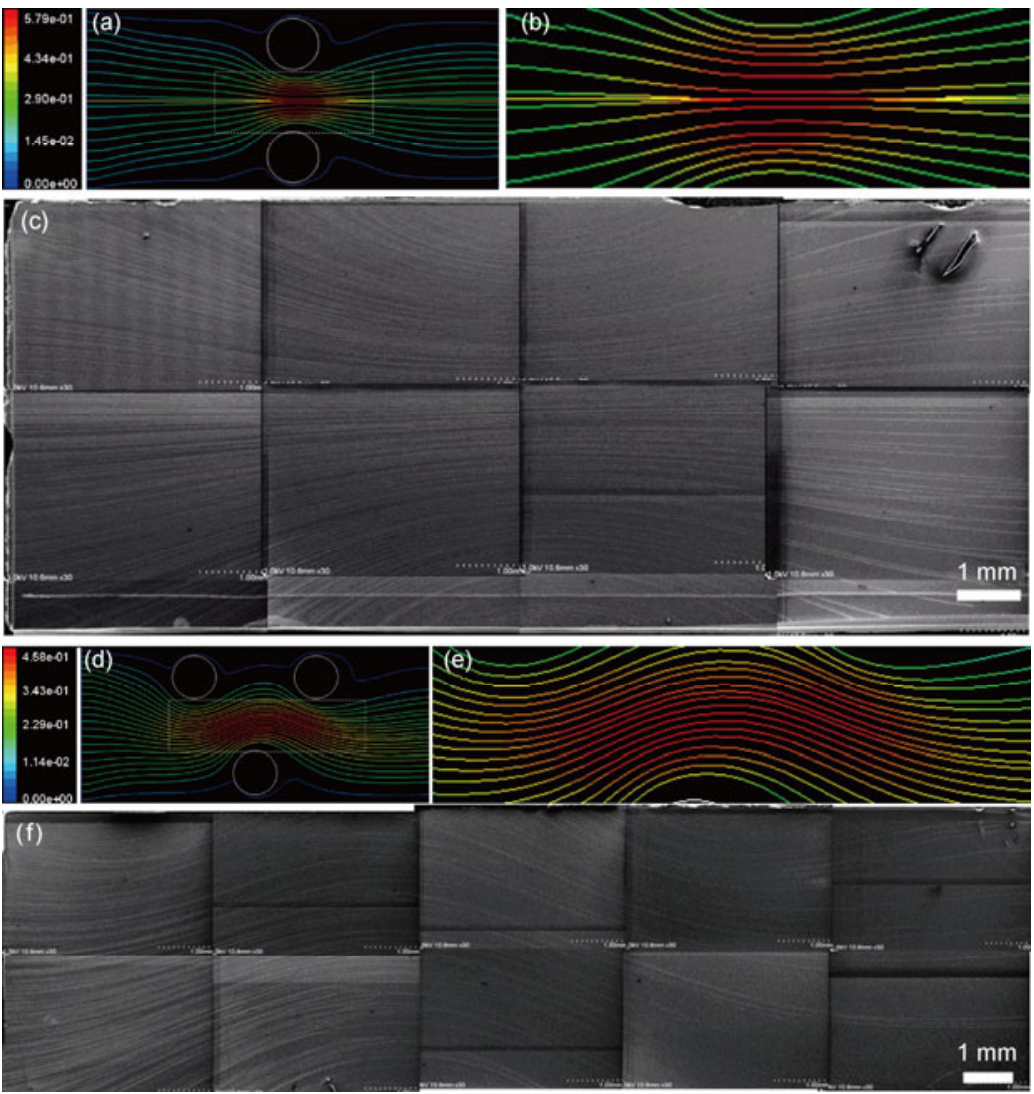

Figure 2 (a) The hydromechanical simulation result when two cylindrical barriers were placed each on one side of the substrate. (b) The magnified image of (a) shows the streamline pattern at the place where the silicon wafer substrate was located. (c) Merged SEM images show the panorama of the obtained SWNTs. (d) The hydromechanical simulation result when three cylindrical barriers were placed on both sides of the substrate. (e) The magnified image of (d) shows the streamline pattern at the place where the silicon wafer substrate was located. (f) Merged SEM images show the panorama of the obtained SWNTs [8].

(a)
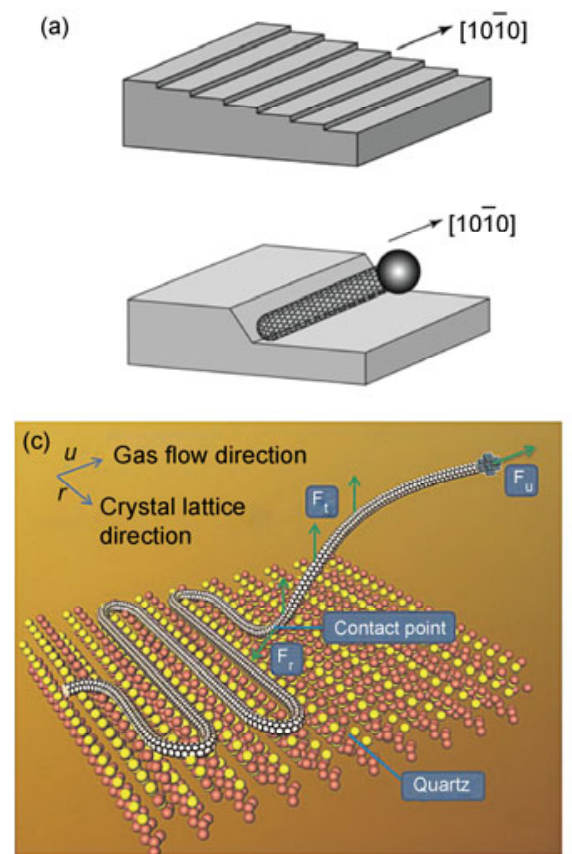
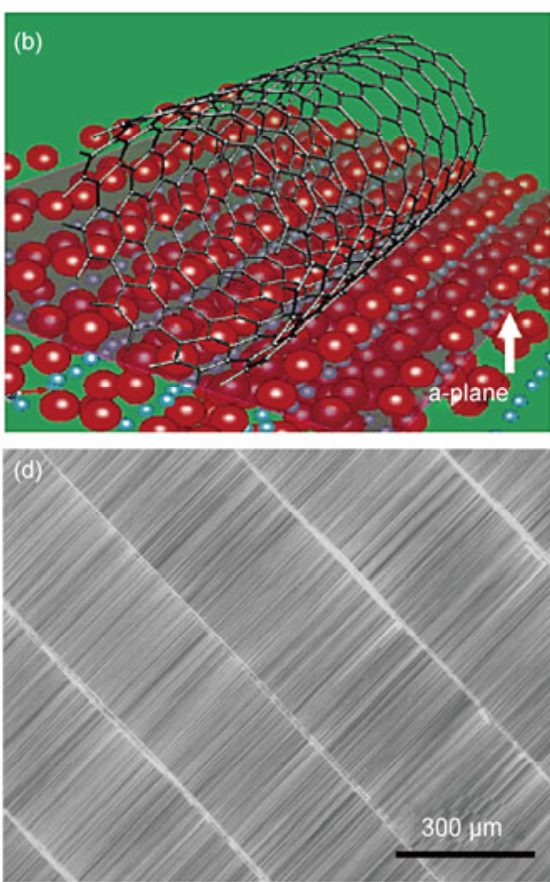

Figure 3 (a) Schematic illustration of the aligning mechanism of nanotubes at the faceted nanostep [9]. (b) Schematic illustration of a SWNT oriented on an a-plane sapphire substrate [10]. (c) Scheme of a serpentine SWNT on quartz [13]. (d) SEM image of SWNT arrays grown on quartz using ethanol as carbon source [14]. 
nanotubes (MWNTs) and SWNT bundles are almost avoided with the substrate oriented growth.

\section{Conductivity and chirality control}

As stated before, SWNTs can be either metallic or semiconducting depending on their structure. The band gap of SWNTs is determined by their chirality. Even though the devices made by individual SWNTs may have good performance, there are still major problems for CNT-based nanoelectronics. The bottleneck is that none of the present methods can directly produce SWNTs of uniform conductivity and identical chirality. Normal methods can only obtain mixtures of metallic and semiconducting SWNTs of different structures. Over the past decade, many groups have devoted to developing methods to separate metallic and semiconducting SWNTs. Electrophoresis, density gradient induced centrifugation, selective elimination by electrical breakdown, gas-phase plasma etching, and several other methods have been developed. However, all of the post-treatment methods introduce defects and contaminants to the SWNTs. There are also methods to selectively grow semiconducting or metallic SWNTs and even SWNTs with very narrow chirality. In theses reports, different catalysts such as Co-Mo, Fe-Co, Fe-Ru, Fe-Ni, Au, Fe-Cu, and Co$\mathrm{Mn}$, have been used to selectively grow SWNTs in bulkform [15-22]. Though separation treatments are not needed for these SWNTs, they still have to be dispersed in solution and patterned on the substrates for device fabrication. Therefore, the structure damage and contamination can still not be avoided. Hence, the direct growth of SWNTs with designed electric property on suitable substrates should be a significant advancement toward their easy integration with existing silicon-based fabrication technology.

High-density arrays of perfectly aligned SWNTs consisting almost exclusively of semiconducting nanotubes were grown on ST-cut quartz substrates (Figure 4) [23]. Cu was used as the catalyst. Ethanol and methanol were used as the carbon source. The growth temperature was $900^{\circ} \mathrm{C}$. When only ethanol was used as carbon source, normal samples of mixed metallic and semiconducting SWNTs were obtained (Figure 5(a)). However, when methanol was added, the content of metallic tubes decreased gradually (Figure 5(b)-(d)). The results obviously indicate that the addition of methanol is crucial for the selective growth of
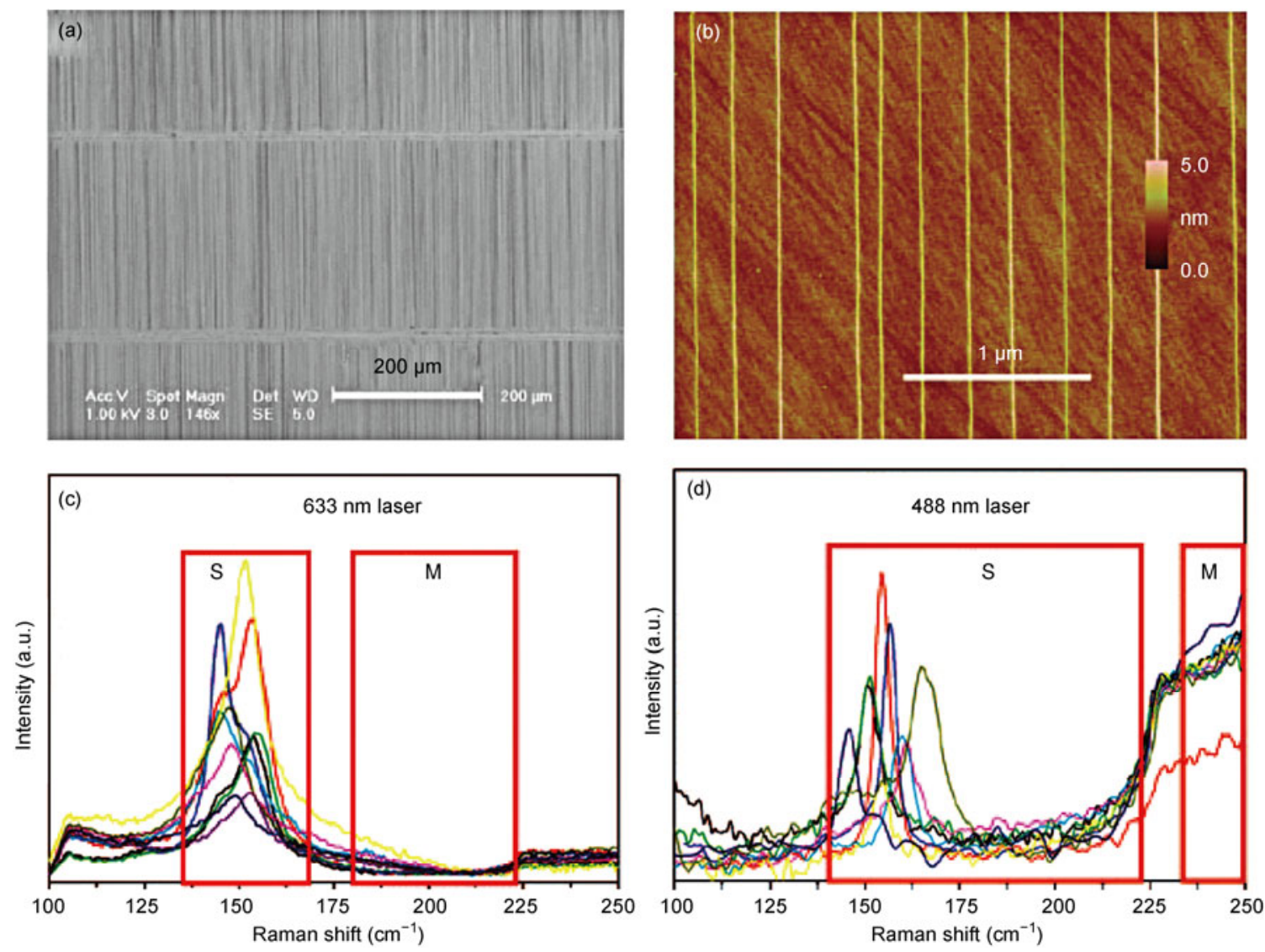

Figure 4 (Color online) SEM image (a) and atomic force microscopy (AFM) image (b) of almost exclusively semiconducting SWNT arrays. (c) and (d) Raman spectra of SWNTs transferred onto the $\mathrm{SiO}_{\mathrm{x}} / \mathrm{Si}$ substrates. The spectra were obtained using 633 and $488 \mathrm{~nm}$ excitation laser lines. Peaks within the rectangles marked with S correspond to the semiconducting SWNTs. The rectangles marked with M denote the frequency range where radial breathing mode (RBM) peaks of metallic SWNTs are expected [23]. 

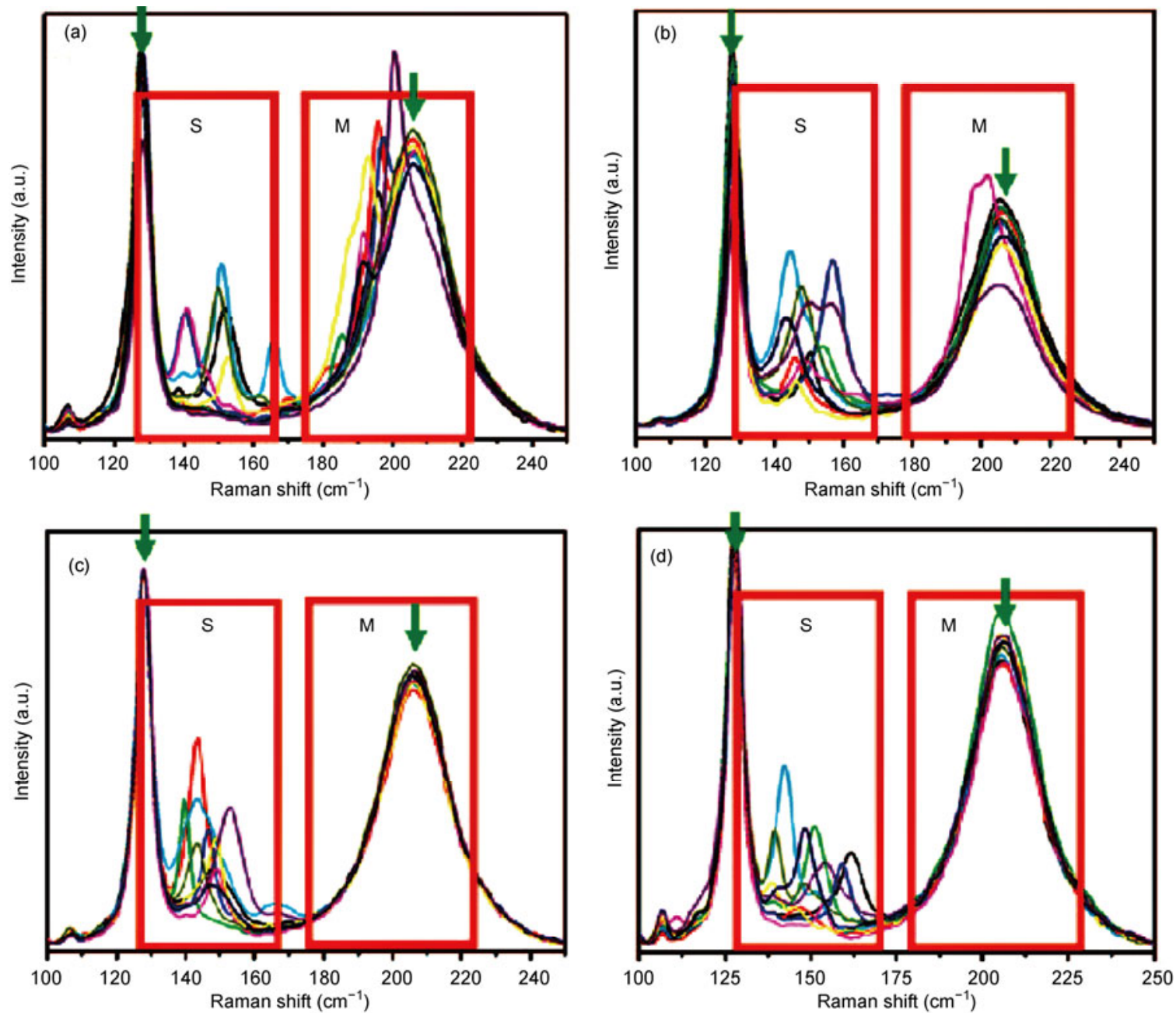

Figure 5 (Color online) Raman spectra showing RBM peaks of as-prepared SWNTs on ST-cut quartz with different methanol/ethanol ratios. The flow rate of ethanol was set as $150 \mathrm{sccm}$, and the flow rate of methanol was (a) $0 \mathrm{sccm}$, (b) $150 \mathrm{sccm}$, (c) $450 \mathrm{sccm}$, and (d) $600 \mathrm{sccm}$, respectively. The sharp peaks at $127 \mathrm{~cm}^{-1}$ and the broad peaks at $205 \mathrm{~cm}^{-1}$, marked with green arrows, correspond to the Raman spectra of the quartz. Peaks marked with $\mathrm{S}$ within the rectangles correspond to the semiconducting SWNTs. The rectangles marked with M denote the frequency range where RBM peaks of metallic SWNTs are expected [23].

semiconducting SWNTs. It is known that methanol can give off $\mathrm{OH}$ radicals at high temperature. Because metallic SWNTs have smaller ionization potential than the semiconducting ones, they should be easier to be oxidized by the $\mathrm{OH}$ radicals. This might be the reason why the presence of methanol in feeding stocks leads to the selective production of semiconducting SWNTs. On the other hand, no selectivity was found on $\mathrm{SiO}_{x} / \mathrm{Si}$ substrates when same CVD condition was used. This indicates that the substrate is another important factor for the selective growth of semiconducting nanotubes. In our previous study, double-walled and triple-walled carbon nanotubes (CNTs) were often found on $\mathrm{SiO}_{x} / \mathrm{Si}$ substrates, but never seen on quartz wafers. MWNTs are much more stable therefore more difficult to be oxidized than SWNTs. Most probably, the formation of MWNTs is the reason why no selectivity of semiconducting CNTs was observed on $\mathrm{SiO}_{x} / \mathrm{Si}$ substrates when methanol was added to the feeding stocks.

Hong et al. [24] introduced UV irradiation to the growth process and directly grew semiconducting nanotubes on quartz. The percentage of semiconducting SWNTs can be higher than $95 \%$. They indicate that the selection process took place at the very beginning of the SWNT formation rather than destroying the metallic SWNTs after growth. They suggested that the SWNTs may be destroyed by UV irradiation through a free radical reaction.

Though semiconducting SWNTs of high purity have already produced, yet there are no methods to realize the controlled growth of SWNTs with designed identical structure. We still have a long way to go for the chirality selective growth of SWNTs.

\section{Catalysts for the growth of SWNTs}

The vapor-liquid-solid (VLS) mechanism is generally accepted by most researchers for the CVD growth of SWNTs. In VLS mechanism, the catalyst particles have two 
important functions during the formation of SWNTs. One is catalyzing the decomposition of the carbon feeding gas and the other is offering an initial centre for CNT nucleation. Therefore, the type, morphology and properties of the catalyst particles play important roles in determining the structure and quality of the SWNTs. Catalyst is an essential factor in the controlled growth of SWNTs [14].

Over the years, $\mathrm{Fe}, \mathrm{Co}$, and $\mathrm{Ni}$ were the most commonly used catalysts for SWNT growth. $\mathrm{Cu}$ was considered not a good catalyst for its low activity to decompose carbon source and low carbon solubility. However, we found that $\mathrm{Cu}$ is really a good catalyst for SWNTs' growth [25,26]. Compared with $\mathrm{Fe}$, the lower activity to decompose carbon source of $\mathrm{Cu}$ is helpful for the preparation of high quality SWNT because less amorphous carbon and MWNTs was formed. SWNTs grew much longer and straighter with $\mathrm{Cu}$ than with $\mathrm{Fe}$ in the fast-heating CVD processes (Figure 6(a) and (b)). The lower activity of $\mathrm{Cu}$ to decompose carbon
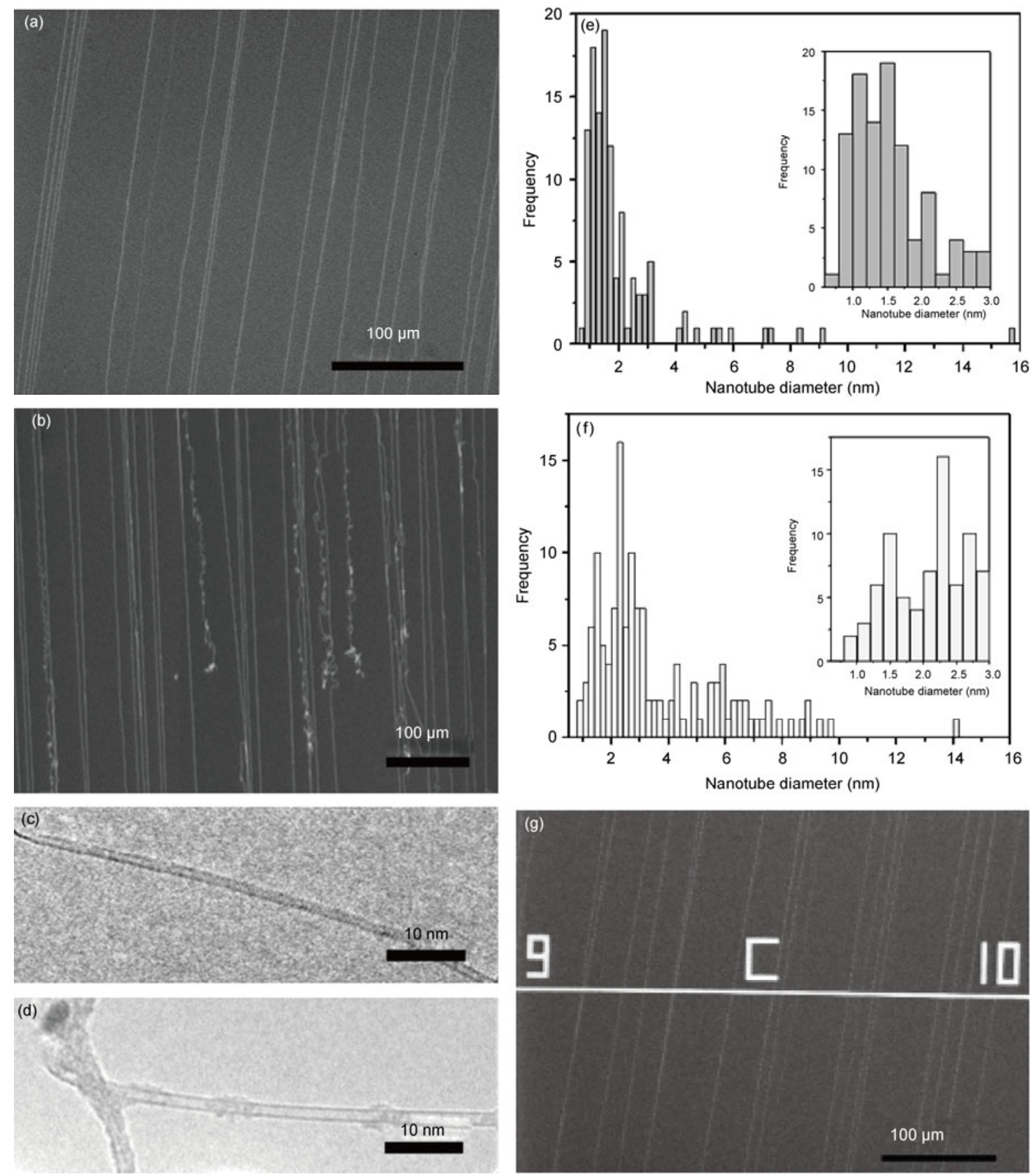

Figure 6 SEM images, high resolution transmission electron microscopy (HRTEM) images, and diameter distribution statistics of SWNT arrays grown by using $\mathrm{Cu}((\mathrm{a}),(\mathrm{c}),(\mathrm{e}))$ and $\mathrm{Fe}((\mathrm{b}),(\mathrm{d}),(\mathrm{f}))$ as catalysts [26]. (g) SEM image of SWNT arrays grown by using Pb as the catalyst [27]. 
source may remarkably reduce the chance of catalyst poisoning by over loading of carbon. Another advantage of $\mathrm{Cu}$ is its weaker interaction with the $\mathrm{SiO}_{x} / \mathrm{Si}$ substrates [26]. This is obviously favorable for lifting up the catalyst particles in the gas flow and the growth of ultralong SWNTs via "kite-mechanism". According to the AFM measurement, the SWNTs prepared by $\mathrm{Cu}$ catalyst show a quite narrow diameter distribution and more than $85 \%$ of the tubes present diameters smaller than $3 \mathrm{~nm}$. The mean diameter is $1.5 \pm 0.5$ nm (Figure 6 (e)). The SWNTs prepared by Fe show a wider diameter distribution and the percentage of the nanotube with the diameter below $3 \mathrm{~nm}$ is only $58 \%$ (Figure 6(f)). The results indicate that most of the SWNTs prepared by $\mathrm{Cu}$ are individually standing SWNTs, and more MWNTs and SWNT bundles are prepared by Fe. The higher quality of SWNTs obtained by $\mathrm{Cu}$ catalysts is beneficial for building electronic devices with better performance.

As the binary phase diagram of $\mathrm{C}-\mathrm{Pb}$ is very similar to that of $\mathrm{C}-\mathrm{Cu}, \mathrm{Pb}$ also shows to be a good catalyst for SWNT growth [27]. The low melting point of $\mathrm{Pb}\left(\sim 327^{\circ} \mathrm{C}\right)$ has two opposite effects on the preparation of SWNTs. The volatility of $\mathrm{Pb}$ makes it easy to obtain SWNTs without any metallic contaminants. This is desired for the application of SWNTs in high performance nanoelectronics and biomedical fields. However, the too high volatility may lead the vanishment of catalyst particles before the nucleation of nanotubes at the high growth temperature of $850-950{ }^{\circ} \mathrm{C}$. Several methods have been designed to maintain metallic $\mathrm{Pb}$ particles at the initial stage of the growth, including low gas flow method, fast-heating method and polyvinylpyrrolidone (PVP) assisted method. Using these methods, we successfully obtained random SWNT networks and horizontally aligned ultralong SWNT arrays (Figure 6(g)). XPS and ICP measurements show that there are no metallic catalyst residuals in these SWNT samples [25,28].

Catalysts play important roles in SWNT growth. Recently, many reports have shown that the composition, size, and structure of the catalyst particles may affect the structure of SWNTs [14-22]. We should put more efforts on understanding the growth mechanism of SWNTs and the design of catalysts. This is important for realizing chirality controlled growth of SWNTs.

\section{Conclusions}

Direct growth of SWNTs on the substrates by CVD process is the most promising approach to obtain SWNTs for high performance nanoelectronic devices. The orientation of the nanotubes can be guided by gas flow and crystalline substrates. Especially, the gas flow method is suitable for growing SWNTs on silicon wafers, which is compatible with the present silicon-based technology. The chirality and conductivity of the SWNTs can be controlled to some extent. More efforts are still needed to fully understand the mechanism of tube growth and the chirality selectivity. This is essential to reach the ultimate goal of obtaining SWNTs with the desired specific chirality. Catalyst is a key factor for the controlled growth of SWNTs. Understanding how catalysts affect the growth process and consequently the rational catalyst design is very important to precisely control the structure and properties of SWNTs. For the commercial application of SWNTs as the materials for next generation electronic devices, accurate control on the position, orientation and structure of SWNTs on substrates is required. There is still a long way to touch the goal.

This work was supported by the National Basic Research Program of China (2011CB933003) and the National Natural Science Foundation of China (11179011 and 21125103).

1 Iijima S, Ichihashi T. Single-shell carbon nanotubes of 1-nm diameter. Nature, 1993, 363: 603-605

2 Saito R D, Dresselhaus M S, Dresselhaus G. Physical Properties of Carbon Nanotubes. Singapore: World Scientific Publishing, 1998

3 Javey A, Guo J, Farmer D B, et al. Self-aligned ballistic molecular transistors and electrically parallel nanotube arrays. Nano Lett, 2004, 4: $1319-1322$

4 Javey A, Tu R, Farmer D B, et al. High performance n-type carbon nanotube field-effect transistors with chemically doped contacts. Nano Lett, 2005, 5: 345-348

5 Zhang Z Y, Liang X L, Wang S, et al. Doping-free fabrication of carbon nanotube based ballistic CMOS devices and circuits. Nano Lett, 2007, 7: 3603-3607

6 Huang S M, Woodson M, Smalley R, et al. Growth mechanism of oriented long single walled carbon nanotubes using "fast-heating" chemical vapor deposition process. Nano Lett, 2004, 4: 1025-1028

7 Jin Z, Chu H B, Wang J Y, et al. Ultralow feeding gas flow guiding growth of large-scale horizontally aligned single-walled carbon nanotube arrays. Nano Lett, 2007, 7: 2073-2079

8 Liu Y, Hong J X, Zhang Y, et al. Flexible orientation control of ultralong single-walled carbon nanotubes by gas flow. Nanotechnology, 2009, 20: 185601

9 Ismach A, Kantorovich D, Joselevich E. Carbon nanotube graphoepitaxy: Highly oriented growth by faceted nanosteps. J Am Chem Soc, 2005, 127: 11554-11555

10 Han S, Liu X L, Zhou C W. Template-free directional growth of single-walled carbon nanotubes on a- and r-plane sapphire. J Am Chem Soc, 2005, 127: 5294-5295

11 Ding L, Yuan D N, Liu J. Growth of high-density parallel arrays of long single-walled carbon nanotubes on quartz substrates. J Am Chem Soc, 2008, 130: 5428

12 Yuan D N, Ding L, Chu H B, et al. Horizontally aligned singlewalled carbon nanotube on quartz from a large variety of metal catalysts. Nano Lett, 2008, 8: 2576-2579

13 Yao Y G, Dai X C, Feng C Q, et al. Crinkling ultralong carbon nanotubes into serpentines by a controlled landing process. Adv Mater, 2009, 21: 4158

14 Li Y, Cui R L, Ding L, et al. How catalysts affect the growth of single-walled carbon nanotubes on substrates. Adv Mater, 2010, 22: 1508-1515

15 Bachilo S M, Balzano L, Herrera J E, et al. Narrow (n,m)-distribution of single-walled carbon nanotubes grown using a solid supported catalyst. J Am Chem Soc, 2003, 125: 11186-11187

16 Chiang W H, Sankaran R M. Linking catalyst composition to chirality distributions of as-grown single-walled carbon nanotubes by tuning NixFe1-x nanoparticles. Nat Mater, 2009, 8: 882-886

17 Ciuparu D, Chen Y, Lim S, et al. Uniform-diameter single-walled carbon nanotubes catalytically grown in cobalt-incorporated MCM- 
41. J Phys Chem B, 2004, 108: 503-507

18 Ghorannevis Z, Kato T, Kaneko T, et al. Narrow-chirality distributed single-walled carbon nanotube growth from nonmagnetic catalyst. J Am Chem Soc, 2010, 132: 9570-9572

19 He M, Chernov A I, Fedotov P V, et al. Predominant (6,5) singlewalled carbon nanotube growth on a copper-promoted iron catalyst. J Am Chem Soc, 2010, 132: 13994-13996

20 Li X L, Tu X M, Zaric S, et al. Selective synthesis combined with chemical separation of single-walled carbon nanotubes for chirality selection. J Am Chem Soc, 2007, 129: 15770

21 Loebick C Z, Podila R, Reppert J, et al. Selective synthesis of subnanometer diameter semiconducting single-walled carbon nanotubes. J Am Chem Soc, 2010, 132: 11125-11131

22 Wang H, Wang B, Quek X Y, et al. Selective synthesis of $(9,8)$ single walled carbon nanotubes on cobalt incorporated TUD-1 catalysts. J Am Chem Soc, 2010, 132: 16747-16749
23 Ding L, Tselev A, Wang J Y, et al. Selective growth of well-aligned semiconducting single-walled carbon nanotubes. Nano Lett, 2009, 9: 800-805

24 Hong G, Zhang B, Peng B H, et al. Direct growth of semiconducting single-walled carbon nanotube array. J Am Chem Soc, 2009, 131: 14642

25 Zhou W W, Han Z Y, Wang J Y, et al. Copper catalyzing growth of single-walled carbon nanotubes on substrates. Nano Lett, 2006, 6: 2987-2990

26 Cui R L, Zhang Y, Wang J Y, et al. Comparison between copper and iron as catalyst for chemical vapor deposition of horizontally aligned ultralong single-walled carbon nanotubes on silicon substrates. J Phys Chemistry C, 2010, 114: 15547-15552

27 Zhang Y, Zhou W W, Jin Z, et al. Direct growth of single-walled carbon nanotubes without metallic residues by using lead as a catalyst. Chem Mater, 2008, 20: 7521-7525

Open Access This article is distributed under the terms of the Creative Commons Attribution License which permits any use, distribution, and reproduction in any medium, provided the original author(s) and source are credited. 